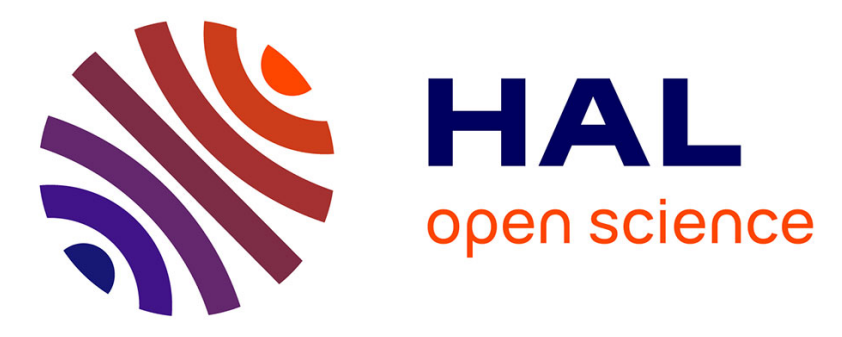

\title{
Higher variational equation techniques for the integrability of homogeneous potentials
}

Thierry Combot

\section{To cite this version:}

Thierry Combot. Higher variational equation techniques for the integrability of homogeneous potentials. Maitine Bergounioux; Gabriel Peyré; Christoph Schnörr; Jean-Baptiste Caillau; Thomas Haberkorn. Variational Methods In Imaging and Geometric Control, 18, De Gruyter, pp.365-386, 2017, Radon Series on Computational and Applied Mathematics, 9783110430394. 10.1515/9783110430394012 . hal-01514112

\section{HAL Id: hal-01514112 \\ https://u-bourgogne.hal.science/hal-01514112}

Submitted on 4 Mar 2021

HAL is a multi-disciplinary open access archive for the deposit and dissemination of scientific research documents, whether they are published or not. The documents may come from teaching and research institutions in France or abroad, or from public or private research centers.
L'archive ouverte pluridisciplinaire HAL, est destinée au dépôt et à la diffusion de documents scientifiques de niveau recherche, publiés ou non, émanant des établissements d'enseignement et de recherche français ou étrangers, des laboratoires publics ou privés. 


\title{
Thierry Combot
}

\section{Higher variational equation techniques for the integrability of homogeneous potentials}

\begin{abstract}
We present several methods using higher variational equations to study the integrability of Hamiltonian systems from the algebraic and computational point of view. Through the Morales Ramis Simo theorem, strong integrability conditions can be computed for Hamiltonian systems, allowing us to prove nonintegrability even for potentials with parameters. This theorem can, in particular, be applied to potentials, even transcendental ones, by properly defining them on complex Riemann surfaces. In the even more particular case of homogeneous potentials, a complete computation of integrability conditions of variational equation near straight line orbits is possible at arbitrary order, allowing us to prove the nonintegrability of certain $n$-body problems which were inaccessible due to the complicated central configuration equation.
\end{abstract}

Keywords: Higher variational equations, differential Galois theory, integrability

AMS classification: $37 \mathrm{~J} 30$

\subsection{Introduction: integrable systems}

In this paper, we consider Hamiltonian systems that are defined by an analytic function $H: \mathcal{M} \longrightarrow \mathbb{C}$ with $\mathcal{M}$ being a complex analytic symplectic manifold. The associated dynamical system is the following differential equation system:

$$
\dot{q}_{i}=\partial_{p_{i}} H, \quad \dot{p}_{i}=-\partial_{q_{i}} H, \quad i=1, \ldots, n .
$$

The dimension of $\mathcal{M}$ is $2 n$, where $n$ is called the number of degrees of freedom. This kind of differential system can sometimes be integrated, that is, the solutions can be "explicitly found." This has led to the following (complex version) notion of integrability.

Definition 1.1 (Arnold [2]). Let $M$ be a complex symplectic manifold of dimension $2 n$ and $H: \mathcal{M} \longrightarrow \mathbb{C}$ a complex analytic Hamiltonian with $n$ degrees of freedom. Assume that there exist $I_{1}=H, \ldots, I_{n}$, with $n$ being analytic functions, and $I_{i}: \mathcal{M} \longrightarrow \mathbb{C}$ such that

- For all $i=1, \ldots, n$, we have $\left\{H, I_{i}\right\}=0$. We say that the functions $I_{i}$ are first integrals.

- For all $i, j=1, \ldots, n,\left\{I_{i}, I_{j}\right\}=0$. We say that the functions $I_{i}$ are in involution. To say that $I_{i}$ is a first integral is equivalent to being in involution with $H=I_{1}$.

Thierry Combot, 6 Avenue Alain Savary, 21000 Dijon, France, thierry.combot@u-bourgogne.fr 
- $\quad$ The Jacobian matrix $A \in M_{n, 2 n}(\mathbb{C})$ given by

$$
\begin{aligned}
A_{i, j} & =\partial_{p_{j}} I_{i} i=1, \ldots, n, j=1, \ldots, n, \\
A_{i, j+n} & =\partial_{q_{j}} I_{i} i=1, \ldots, n, j=1, \ldots, n,
\end{aligned}
$$

has a full rank on an open dense set of $\mathcal{M}$. This property is called independent nearly everywhere.

Then, $H$ is called Liouville integrable.

An important consequence of integrability is solvability by quadrature. For real systems, that is, real analytic Hamiltonians $H: \mathcal{M} \longrightarrow \mathbb{R}$, with $\mathcal{M}$, analytic symplectic and real first integrals, some dynamical consequences follows from integrability.

Theorem 1.2 (Arnold-Liouville-Mineur [2]). Let $\mathcal{M}$ be a symplectic manifold of dimension $2 n$ and $H: \mathcal{M} \longrightarrow \mathbb{R}$ an analytic Hamiltonian. If $H$ is Liouville integrable with real first integrals $I_{1}, \ldots, I_{n}$, then for any level set

$$
\mathcal{M}_{f}=\left\{(p, q) \in \mathcal{M}: I_{i}(p, q)=f_{i}, i=1, \ldots, n\right\}
$$

such that $I_{1}, \ldots, I_{n}$ are independent of $\mathcal{M}_{f}$, and we have

- $\mathcal{M}_{f}$ is a smooth manifold, invariant by the flow of $H$.

- If $\mathcal{M}_{f}$ is compact connected, then it is diffeomorphic to the n-dimensional torus

$$
\mathbb{T}^{n}=\left\{\left(\varphi_{1}, \ldots, \varphi_{n}\right) \bmod 2 \pi\right\}
$$

- If $\mathcal{M}_{f}$ is compact connected, the Hamiltonian flow of $H$ on $\mathcal{M}_{f}$ is quasiperiodic.

Integrable Hamiltonians are very rare, but they are very useful for understanding the system quantitatively, like the behavior of solutions, stability. The fundamental problem of knowing the integrability of a system is to find the functions $I_{i}$, on which we know nothing a priori. We typically assume that they belong to some class of functions (in the following rational), but nothing can be said about their degree for example.

In the following, we will consider rational first integral, that is, rational functions on algebraic manifolds $\mathcal{M}$, as we will focus on this problem from a computer algebra point of view. Remark that all algebraic manifolds $\mathcal{M}$ are not equivalent to $\mathbb{C}^{2 n}$, and thus rational functions on such manifolds cannot always be represented as $2 n$ variables' rational functions.

\subsection{An algebraic point of view}

\subsubsection{Algebraic presentation of a Hamiltonian system}

In this paper, we will focus on integrability from an algebraic point of view. The Hamiltonian is assumed to be rational on $\mathcal{M}$, and the complex manifold itself will typically 
be algebraic. The manifold $\mathcal{M}$ is, thus, typically defined by $k$ algebraic equations on $\mathbb{C}^{2 n+k}$. This manifold can be projected as

$$
\pi: \mathcal{M} \longrightarrow \mathbb{C}^{2 n},
$$

where $\mathbb{C}^{2 n}$ is the space of canonical variables $p, q$, but then a rational function $f$ on $\mathcal{M}$ can appear multivalued as a function of $p, q$. This is due to the fact that the projection $\pi$ is typically not invertible, and so above each $(p, q) \in \mathbb{C}^{2 n}$ lies several points of $\mathcal{M}$ and so several values of $f$. Said otherwise, the field of rational functions on $\mathcal{M}$ as functions of $p, q$ forms a field extension of $\mathbb{C}(p, q)$. The projection also carries a differentiation structure:

Definition 2.1. A differential field extension $K$ over $\mathbb{C}(p, q)$ is a field $K \supset \mathbb{C}(p, q)$ equipped with $2 n$ differentiations $\partial_{p_{i}}$, $\partial_{q_{i}}$ pairwise commuting and which is stable by the differentiations.

Notation: In the following, the polynomial ideal generated by $P_{1}, \ldots, P_{k} \in \mathbb{C}\left[x_{1}, \ldots\right.$, $\left.x_{n}\right]$ will be noted, $I=\left\langle P_{1}, \ldots, P_{k}\right\rangle$. The common zero level of the elements of $I$ will be noted, $\mathcal{V}(I) \subset \mathbb{C}^{n}$.

The simplest example of differential field is $\mathbb{C}(p, q)$ itself. For more complicated fields, the differentiations need to be defined more carefully, as in the differential field of Example $1, K=\operatorname{Frac}\left(\mathbb{C}\left[p_{1}, p_{2}, q_{1}, q_{2}, s\right] /\left\langle s^{2}-q_{1}^{2}-q_{2}^{2}\right\rangle\right)$. This is a field extension of $\mathbb{C}(p, q)$, and elements of this field are represented by a five-variable rational function $f$. The derivations in $p, q$ are then defined by

$$
\partial_{p_{1}}=\partial_{1}, \quad \partial_{p_{2}}=\partial_{2}, \quad \partial_{q_{1}}=\partial_{3}+\frac{2 q_{1}}{2 s} \partial_{5}, \quad \partial_{q_{2}}=\partial_{4}+\frac{2 q_{2}}{2 s} \partial_{5} .
$$

The notation $\partial_{i}$ means that the representing function $f$ is derived with respect to the $i$-th variable. In our Hamiltonian setting, the differentiations $\partial_{p}, \partial_{q}$ also define the Poisson bracket and, thus, the symplectic form.

If the manifold is algebraic, such differential extensions will always be finite. Typical problems about integrable systems are presented in the other way. The Hamiltonian is given as a multivalued function of $p, q$, and the first step is to find a manifold on which to define it properly. In the case of a algebraic Hamiltonian, we will see in the following examples, this can be made more or less automatically through Groebner basis and polynomial ideal methods.

\section{Some examples}

Example 1. The Hamiltonian of the Keplerian motion

$$
H=\frac{1}{2}\left(p_{1}^{2}+p_{2}^{2}\right)+\frac{1}{\sqrt{q_{1}^{2}+q_{2}^{2}}} .
$$


This expression is well defined in the real domain, but we need to extend it to the complex domain. However, this Hamiltonian is multivalued on $\mathbb{C}^{4}$ : we cannot choose a valuation for the square root, after a loop $\sqrt{q_{1}^{2}+q_{2}^{2}}$ can become $-\sqrt{q_{1}^{2}+q_{2}^{2}}$. To properly define this Hamiltonian on the complex, it is, therefore, necessary to introduce the algebraic extension $q_{1}^{2}+q_{2}^{2}=s^{2}$ and the associated manifold

$$
\mathcal{M}=\left\{(p, q, s) \in \mathbb{C}^{2} \times \mathbb{C}^{2} \times \mathbb{C}: s^{2}=q_{1}^{2}+q_{2}^{2}, s \neq 0\right\} .
$$

The canonical projection $\pi ; \mathcal{M} \longrightarrow \mathbb{C}^{4}$ over variables $(p, q)$ produces from the rational functions on $\mathcal{M}$ the function field $K=\mathbb{C}\left(p, q, \sqrt{q_{1}^{2}+q_{2}^{2}}\right)$ which contains the Hamiltonian.

Remark that the point $s=0$ has been removed to obtain a smooth manifold $M$. This is related to the fact that not only $s=0$ is a singularity of $H$, but also the projection $\pi$ is not good at these points as two branches cross here. This could have consequences for the application of Morales Ramis Simo Theorem as some particular orbits can be removed from the phase space by this process. Here, however, $s=0$ corresponds to a singularity of $H$ anyway. Under this representation, we can note the field

$$
K=\operatorname{Frac}\left(\mathbb{C}[p, q, s] /\left\langle s^{2}-q_{1}^{2}-q_{2}^{2}\right\rangle\right),
$$

where Frac means that we take the fraction field with the derivations

$$
\begin{gathered}
\partial_{p_{1}}=\partial_{1}, \quad \partial_{p_{2}}=\partial_{2}, \quad \partial_{q_{1}}=\partial_{3}+\frac{2 q_{1}}{2 s} \partial_{5}, \quad \partial_{q_{2}}=\partial_{4}+\frac{2 q_{2}}{2 s} \partial_{5}, \\
H=\frac{1}{2}\left(p_{1}^{2}+p_{2}^{2}\right)+\frac{1}{s} .
\end{gathered}
$$

The Hamiltonian is rational on $M$ (as it belongs to $K$ ), and the symplectic form and Poisson bracket are defined through the differentiations given above. In the following, the first integrals of this Hamiltonian will be searched as rational functions on $M$, that is, elements in $K$.

Example 2. Consider the following potential

$$
V\left(q_{1}, q_{2}, w\right)=w^{5}+q_{2}^{2}, \quad I=\left\langle w^{2}-q_{1}\right\rangle
$$

associated with the Hamiltonian $H=\frac{1}{2}\left(p_{1}^{2}+p_{2}^{2}\right)+V(q, w)$. The associated Hamiltonian system has a particular solution given by $w(t)=0, q_{1}(t)=0, q_{2}(t)=\cos t$. However, to properly define the analytical symplectic manifold $M$, we need to remove the locus $w=q_{1}=0$ :

$$
\mathcal{M}=\left\{(p, q, w) \in \mathbb{C}^{3}: w^{2}=q_{1}, w \neq 0\right\} .
$$

This is due to the associated differentiations becoming singular at $w=0$. 


\section{Generalization to transcendental cases}

For a transcendental Hamiltonian, a similar construction of a complex analytic manifold $\mathcal{M}$ (instead of algebraic) can also be done. The manifold is now defined by $k$ holomorphic functions on $\mathbb{C}^{2 n+k}$, and a rational function on $\mathcal{M}$ is simply a restriction of a rational function on $\mathbb{C}^{2 n+k}$ to $\mathcal{M}$. However, in contrary to the algebraic case, to find a set of $k$ holomorphic functions leading to a smooth complex manifold with $H$ rational on it can be difficult [8]. The difficult point here is that we cannot rely on Groebner basis to prove that $\mathcal{M}$ is smooth.

Example 3. A transcendental case,

$$
H=\frac{1}{2} p_{1}^{2}+\cos \left(q_{1}\right)
$$

Although cos is an entire function and, thus, $H$ is well defined on $\mathbb{C}^{2}$, this is a transcendental function. This system can, however, be defined on the manifold

$$
\mathcal{M}=\left\{\left(p_{1}, q_{1}, c, s\right) \in \mathbb{C}^{4}: \quad c=\cos \left(q_{1}\right), s=\sin \left(q_{1}\right)\right\},
$$

on which the Hamiltonian is rational, $H=\frac{1}{2} p_{1}^{2}+c$. The associated differential field is then

$$
K=\operatorname{Frac}\left(\mathbb{C}\left[p_{1}, q_{1}, c, s\right] /\left\langle c^{2}+s^{2}-1\right\rangle\right), \quad \partial_{p_{1}}=\partial_{1}, \partial_{q_{1}}=\partial_{2}-s \partial_{3}+c \partial_{4} .
$$

\section{Parametrization approach}

Another way of obtaining a rational presentation of these Hamiltonians is rational parametrization. If we can find a (symplectic) variable change such that the Hamiltonian becomes rational in the new variables, then we come down to study a rational Hamiltonian on $\mathbb{C}^{2 n}$. In the first two examples we shown, a birational variable change can be made as the manifolds $s^{2}=q_{1}^{2}+q_{2}^{2}$ and $w^{2}-q_{1}=0$ are rationally parametrizable. In Example 1, we have

$$
\begin{aligned}
& \operatorname{Frac}\left(\mathbb{C}[q, s] /\left\langle s^{2}-q_{1}^{2}-q_{2}^{2}\right\rangle\right) \simeq \mathbb{C}(r, z) \text { with } \\
& q_{1}=\frac{r}{2}\left(z+\frac{1}{z}\right), q_{2}=\frac{r}{2 i}\left(z-\frac{1}{z}\right), s=r .
\end{aligned}
$$

We also need to change the variables $p_{1}, p_{2}$ to obtain a symplectic variable change. With this variable change, the Hamiltonian is in the new coordinates

$$
H\left(p_{r}, p_{z}, r, z\right)=\frac{1}{2}\left(p_{r}^{2}-\frac{r^{2} p_{z}^{2}}{z^{2}}\right)+\frac{1}{r}
$$

and, thus, rational on $\mathbb{C}^{4}$.

In Example 3, recalling that this Hamiltonian corresponds, in fact, to a pendulum and that $q$ represents the angle, we can note

$$
\cos q=\frac{1}{2}\left(z+\frac{1}{z}\right) \quad p=-i \frac{w}{z} \quad H(w, z)=-\frac{1}{2} \frac{w^{2}}{z^{2}}+\frac{1}{2}\left(z+\frac{1}{z}\right)
$$


and now the Hamiltonian is rational and properly defined on $\mathbb{C}^{2}$.

The common property of these systems which allow such a parametrization is that the configuration space is rationally parametrizable:

- Example 1 is a potential on a (complex) cone

- Example 2 is a potential on a parabola

- Example 3 is a potential on a circle.

This method, however, requires the parametrization that is difficult and not always possible. The following Hamiltonian

$$
H=\frac{1}{2} p^{2}+\sqrt{1-q^{3}}
$$

is defined on an elliptic curve and, thus, not rationally parametrizable. The process considering the algebraic extension $w^{2}=1-q^{3}$ is not avoidable.

\section{Generalization to algebraic potentials}

This process can be generalized in the case of algebraic potentials [5]. We consider polynomials $G_{1}, \ldots, G_{s} \in \mathbb{C}\left[q_{1}, \ldots, q_{n}, w_{1}, \ldots, w_{s}\right]$ and the ideal $I=\left\langle G_{1}, \ldots, G_{s}\right\rangle$. We assume that $I$ is a prime ideal and that the matrix

$$
J \in M_{s}(\mathbb{C}[q, w]), \quad J_{i, j}=\frac{\partial G_{i}}{\partial w_{j}}, \quad i, j=1, \ldots, s,
$$

has a nonzero determinant modulo the ideal $I$. We define the associated manifold $\mathcal{S}=$ $I^{-1}(0)$ and $\pi: \mathcal{S} \longrightarrow \mathbb{C}^{n}$ the projection on variables $q$.

Let us now define derivations on $\mathcal{S}$. We first introduce the set

$$
\Sigma(I)=\{(q, w) \in \mathcal{S}: \operatorname{det}(J)(q, w)=0\} .
$$

This set will be called the critical set and corresponds to points on $S$ where the Jacobian matrix $J$ of the application $(q, w) \longrightarrow\left(G_{1}, \ldots, G_{S}\right)$ is not invertible. In equation (2.1), we have, in particular,

$$
\Sigma(I)=\left\{(q, s) \in \mathbb{C}^{2} \times \mathbb{C}: s=0, q_{1} \pm i q_{2}=0\right\} .
$$

Remark that this set is at least of codimension 1 because the determinant is not zero modulo $I$. The manifold $\mathcal{S}$ is of dimension $n$, as it is the common zero of $s$ functionally independent $(\operatorname{det}(J) \neq 0)$ polynomials in dimension $n+s$.

Let $U$ be a nonempty open set of $\mathcal{S}$ and $f$ a rational function on $U$. We may now define

$$
\partial_{q_{k}}=\partial_{k} f-\left(\partial_{n+1} f, \ldots, \partial_{n+s} f\right) J^{-1}\left(\partial_{k} G_{1}, \ldots, \partial_{k} G_{s}\right)^{\top},
$$

where $\partial_{i}$ denotes the derivative according to the $i$-th variable (the variables are $q_{1}, \ldots, q_{n}, w_{1}, \ldots, w_{s}$ in this order). These derivatives are well defined outside $\Sigma(I)$. 
Recall that a rational function on a complex algebraic manifold $\mathcal{S}$ is not, strictly speaking, a function, as it has singularities, and even indeterminate points, as, for example,

$$
V(x, y)=\frac{x y}{x^{2}+y^{2}}
$$

which is indeterminate at $(0,0)$. Noting $\Sigma_{0}$ this as the set of indeterminate points of $V$, we now define the critical set of $V$,

$$
\Sigma(V)=\Sigma_{0} \cup(\Sigma(I) \cap U) .
$$

Definition 2.2. A rational potential $V$ on an open set $U \subset \mathcal{S}$ defines the following dynamical system on $\mathbb{C}^{n} \times(U \backslash \Sigma(V))$ :

$$
\dot{q}_{i}=p_{i}, \quad \dot{p}_{i}=-\partial_{q_{i}} V, \quad i=1, \ldots, n \quad \dot{w}_{i}=\sum_{j=1}^{s} \dot{q}_{j} \partial_{q_{j}} w_{i}, \quad i=1, \ldots, s .
$$

Let us remark now that any algebraic potential fits this definition. Consider an algebraic function $V$ on $\mathbb{C}^{n}$ and $P \in \mathbb{C}\left[q_{1}, \ldots, q_{n}\right][w]$ being a nonzero irreducible polynomial such that $P(V(q))=0$. Now, we will define our dynamical system using the $2 n+1$ variables $p, q, w$. The ideal $I=\langle P\rangle$ on $\mathbb{C}\left[q_{1}, \ldots, q_{n}, w\right]$ is prime because $P$ is irreducible. The matrix $J$ is $1 \times 1$ and its determinant is $\partial_{2 n+1} P$. As $P$ is a nonzero irreducible polynomial, we have $\partial_{2 n+1} P \neq 0 \bmod I$; otherwise, $P$ divides $\partial_{2 n+1} P$, which is impossible because $\partial_{2 n+1} P$ is nonzero and of degree lower than $P$. Thus, $\mathcal{S}=\mathcal{V}(I)$ is a manifold of dimension $n$, and $V$ is a rational potential on $\mathcal{S}$, with $V(q, w)=w$.

\subsubsection{First-order variational equations}

Definition 2.3. Let $\dot{x}=X(x)$ be a differential system on an analytic manifold $M$ and $X$ analytic on $M$. Let $\Gamma=\{x(t), t \in \mathbb{C}\}$ be an orbit of $X$. The first-order variational equation is given by

$$
\dot{\xi}=\nabla X(x(t)) \cdot \xi
$$

The first-order variational equation is a linear differential equation with time-dependent coefficients. In our algebraic presentation, the field $X$ is a rational Hamiltonian field, and $\Gamma$ an algebraic curve, that is, a one-dimensional prime ideal $I$ of the polynomial ring associated with the definition field $K$ of $H$.

\section{Some examples}

Example 1. The algebraic potential

$$
V\left(q_{1}, q_{2}, w\right)=w^{5}+q_{2}^{2} \quad I=\left\langle w^{2}-q_{1}\right\rangle
$$

associated with $H=\frac{1}{2}\left(p_{1}^{2}+p_{2}^{2}\right)+V(q, w)$. The associated Hamiltonian system has a particular solution given by $w(t)=0, q_{1}(t)=0, q_{2}(t)=\cos t$. So the curve $\Gamma$ is 
algebraic, given by

$$
\Gamma=\mathcal{V}\left(\left\langle q_{1}, p_{1}, p_{2}^{2}+2 q_{2}^{2}-2\right\rangle\right) .
$$

Remark now that the time parametrization is not important for us: indeed, the existence of first integrals is independent of the parametrization chosen. Thus, any parametrization can be chosen, and in practice, if possible, a parametrization leading to a variational equation with rational coefficients is chosen. Here, we obtain

$$
\dot{\xi}=\left(\begin{array}{cccc}
0 & 0 & 1 & 0 \\
0 & 0 & 0 & 1 \\
-2 & 0 & 0 & 0 \\
0 & 0 & 0 & 0
\end{array}\right) \xi
$$

which can be rewritten as a second-order differential equation

$$
\ddot{\xi}=\left(\begin{array}{cc}
-2 & 0 \\
0 & 0
\end{array}\right) \xi
$$

and with $q_{2}$ parametrization

$$
\left(1-q_{2}^{2}\right) \partial_{q_{2}}^{2} \xi-q_{2} \partial_{q_{2}} \xi=\left(\begin{array}{cc}
-2 & 0 \\
0 & 0
\end{array}\right) \xi
$$

Remark that, in principle, in the second case, the coefficients will always be algebraic, contrary to the first one where the coefficients belong a priori to $\mathbb{C}(p(t), q(t))$ (and, thus, typically are transcendental functions).

In the potential case, the variational equation admits a nice representation as a second-order differential equation as the Hamiltonian system can rewrite itself $\ddot{q}=$ $-\nabla V(q)$.

Example 2. The spatial pendulum

The spatial coordinates $\left(q_{1}, q_{2}, q_{3}\right)$ of the pendulum are restricted to the unit sphere. And the momentum coordinates, thus, have to be restricted to the tangent plane of the sphere at $\left(q_{1}, q_{2}, q_{3}\right)$. After a projection on the $q_{1}, q_{2}$ plane, the potential can be written as

$$
V\left(q_{1}, q_{2}\right)=\sqrt{1-q_{1}^{2}-q_{2}^{2}} \text {. }
$$

Using our previous method, we obtain the following Hamiltonian and symplectic manifold:

$$
\begin{gathered}
V\left(q_{1}, q_{2}, q_{3}\right)=q_{3}, \quad H=\frac{1}{2}\left(p_{1}^{2}+p_{2}^{2}+p_{3}^{2}\right)+q_{3}, \\
\mathcal{M}=\left\{\left(p_{1}, p_{2}, p_{3}, q_{1}, q_{2}, q_{3}\right): q_{1}^{2}+q_{2}^{2}+q_{3}^{2}=1, p_{1} q_{1}+p_{2} q_{2}+p_{3} q_{3}=0\right\} .
\end{gathered}
$$

Now, to study algebraically this system, we will have to consider rational functions on $\mathcal{M}$, which admit the following representation in the variables $p_{1}, p_{2}, p_{3}, q_{1}, q_{2}, q_{3}$ :

$$
K=\operatorname{Frac}\left(\mathbb{C}[p, q] /\left\langle q_{1}^{2}+q_{2}^{2}+q_{3}^{2}-1, p_{1} q_{1}+p_{2} q_{2}+p_{3} q_{3}\right\rangle\right)
$$


with the derivations

$$
\begin{gathered}
\partial_{q_{1}}=\partial_{4}-\frac{q_{1}}{q_{3}} \partial_{6}-\left(\frac{p_{1}}{q_{3}}+\frac{\left(p_{1} q_{1}+p_{2} q_{2}\right) q_{1}}{q_{3}^{3}}\right) \partial_{3}, \\
\partial_{q_{2}}=\partial_{5}-\frac{q_{2}}{q_{3}} \partial_{6}-\left(\frac{p_{2}}{q_{3}}+\frac{\left(p_{1} q_{1}+p_{2} q_{2}\right) q_{2}}{q_{3}^{3}}\right) \partial_{3}, \\
\partial_{p_{1}}=\partial_{1}-\frac{q_{1}}{q_{3}} \partial_{3}, \quad \partial_{p_{2}}=\partial_{2}-\frac{q_{2}}{q_{3}} \partial_{3} .
\end{gathered}
$$

This system is a two degrees of freedom Hamiltonian. Let us consider a circular motion

$$
\Gamma=\left\{q_{3}=-1 / 2, p_{3}=0\right\} .
$$

The Jacobian of the Hamiltonian field is computed modulo the ideal

$$
\mathcal{J}=\left\langle q_{1}^{2}+q_{2}^{2}+q_{3}^{2}-1, p_{1} q_{1}+p_{2} q_{2}+p_{3} q_{3}, 2 q_{3}+1, p_{3}\right\rangle .
$$

Direct computation of the Jacobian using the derivations formulas gives a matrix with coefficients $\mathbb{C}(p, q)$. Then, each entry is reduced by the following Groebner basis approach:

- An entry fraction $P / Q$ is written as the ideal $\mathcal{J}+\langle f Q-P\rangle$.

- A Groebner basis is computed in lexicographic order with $f$ being the dominant variable. The smallest element $e$ (with respect to the monomial order) of the basis containing the $f$ variable is chosen.

- Solving $e$ in $f$ gives a new rational fraction, which is "simplified" in the normal form.

The matrix obtained is

$$
\left(\begin{array}{cccc}
-4 q_{2} p_{2} & 4 p_{2} q_{1} & -4 q_{2}^{2}+4 & 4 q_{1} q_{2} \\
4 p_{1} q_{2} & 4 q_{2} p_{2} & 4 q_{1} q_{2} & 4 q_{2}^{2}+1 \\
-4 p_{1}^{2}+8 q_{2}^{2}-8 & -4 p_{1} p_{2}-8 q_{1} q_{2} & 4 q_{2} p_{2} & -4 p_{1} q_{2} \\
-4 p_{1} p_{2}-8 q_{1} q_{2} & -4 p_{2}^{2}-8 q_{2}^{2}-2 & -4 p_{2} q_{1} & -4 q_{2} p_{2}
\end{array}\right)
$$

Now comes the parametrization of the curve $\Gamma$. The time parametrization is given by

$$
\sqrt{3} / 2 \cos (t \sqrt{2}), \sqrt{3} / 2 \sin (t \sqrt{2}),-\sqrt{6} / 2 \sin (t \sqrt{2}), \sqrt{6} / 2 \cos (t \sqrt{2}),
$$

which leads to a variational equation with transcendental coefficients. A rational variational equation can be obtained through a rational parametrization of $\Gamma$ by noting

$$
\cos (t \sqrt{2})=\frac{1}{2}\left(z+\frac{1}{z}\right) \quad \sin (t \sqrt{2})=\frac{1}{2 i}\left(z-\frac{1}{z}\right) .
$$

The matrix of the variational equation after this parametrization is given by

$$
\left(\begin{array}{cccc}
\frac{3\left(z^{4}-1\right)}{4 z^{3}} & -\frac{3 i\left(z^{2}+1\right)^{2}}{4 z^{3}} & -\frac{i \sqrt{2}\left(3 z^{4}+10 z^{2}+3\right)}{8 z^{3}} & -\frac{3 \sqrt{2}\left(z^{4}-1\right)}{8 z^{3}} \\
-\frac{3 i\left(z^{2}-1\right)^{2}}{4 z^{3}} & -\frac{3\left(z^{4}-1\right)}{4 z^{3}} & -\frac{3 \sqrt{2}\left(z^{4}-1\right)}{8 z^{3}} & \frac{i \sqrt{2}\left(3 z^{4}-10 z^{2}+3\right)}{8 z^{3}} \\
\frac{4 i \sqrt{2}}{z} & 0 & -\frac{3\left(z^{4}-1\right)}{4 z^{3}} & \frac{3 i\left(z^{2}-1\right)^{2}}{4 z^{3}} \\
0 & \frac{4 i \sqrt{2}}{z} & \frac{3 i\left(z^{2}+1\right)^{2}}{4 z^{3}} & \frac{3\left(z^{4}-1\right)}{4 z^{3}}
\end{array}\right) .
$$


Remark that in the case where the curve $\Gamma$ can be rationally parametrized, it is always possible to obtain a variational equation with rational coefficients. This property will be important for the further study of the differential Galois group of the variational equation. In the nonparametrizable case, it can still be possible that a good parametrization allows us to obtain a variational equation with rational coefficients, as in the case of homogeneous potentials.

Let us consider $V \in \mathbb{C}\left(q_{1}, \ldots, q_{n}\right)$ a homogeneous function of homogeneity degree $k \neq 0$.

Definition 2.4. A point $c \in \mathbb{C}^{n} \backslash\{0\}$ such that

$$
\partial_{q_{1}} V(c)=k c_{1} \quad \cdots \quad \partial_{q_{n}} V(c)=k c_{n}
$$

is a called a Darboux point of $V$. The number $k$ on the right-hand side of the equations is called the multiplier associated with $c$.

In the definition of Darboux points, the multiplier can be fixed arbitrary to any nonzero value, and here we have chosen $k$. We now consider the curve $\Gamma \subset \mathbb{C}^{2 n}$ given by

$$
q(t)=c \phi(t), \quad p(t)=c \cdot \dot{\phi}(t), \quad \frac{1}{2} \dot{\phi}^{2}=-\phi^{k}+1,
$$

where $c$ is a Darboux point of $V$ with multiplier $k$. This curve $\Gamma$ is an orbit of $V$.

The important point here is that to each Darboux point, we can associate an algebraic orbit of $V$. As the Darboux point equation is an algebraic equation with equal number of unknowns and equations, such a point generically exists, and, thus, such an orbit generically exists for homogeneous potentials.

Let us now focus on the type of curve $\Gamma$. Due to the equation $\frac{1}{2} \dot{\phi}^{2}=-\phi^{k}+1$, the curve is typically a hyperelliptic curve (except for some small values of $k$ ), and thus not rationally parametrizable:

$$
\ddot{X}=-\phi(t)^{k-2} \nabla^{2} V(c) X .
$$

Still, another miracle occurs: the parametrization of the curve in $\phi^{k}$ leads to a rational variational equation. This was first noted by Yoshida [23]. The system can furthermore be uncoupled through the diagonalization of the Hessian (in the generic case), leading to $n$-uncoupled second-order variational equations

$$
z(z-1) \ddot{X}_{i}+\left(\frac{3 k-2}{2 k} z-\frac{k-1}{k}\right) \dot{X}_{i}-\frac{\lambda_{i}}{2 k^{2}} X_{i}=0, \text { where } \quad \lambda_{i} \in \operatorname{Spect}\left(\nabla^{2} V(c)\right) .
$$

\subsubsection{Differential Galois theory}

Let us consider a linear differential equation

$$
\dot{X}=A(t) X \quad \text {, where } A \in \mathbb{C}(t) \text {. }
$$


We are interested in the first integrals of this system, that is, rational functions in $X, t$ which are constant with respect to time. The purpose of differential Galois theory is even larger: to determine all algebraic relations between the solutions of these differential equations.

The solutions of equations (2.6) can be formally written by the fundamental matrix $R(t)$, such that

$$
\dot{R}=A R, \quad R(0)=I_{n} .
$$

Let us consider the polynomial ring $K=\mathbb{C}(t)\left[x_{1,1}, \ldots, x_{n, n}, d\right]$ of $n^{2}+1$ variables. We will see an element of this ring as a polynomial function with coefficients in $\mathbb{C}(t)$, taking as arguments a matrix

$$
\left(\begin{array}{lll}
x_{1,1} & \ldots & x_{1, n} \\
& \cdots & \\
x_{n, 1} & \cdots & x_{n, n}
\end{array}\right)
$$

and a complex number $d$. We consider the following ideal:

$$
\operatorname{Inv}=\left\{P \in K: P\left(R(t), \operatorname{det}(R(t))^{-1}\right)=0\right\} .
$$

This is a polynomial ideal generated by the algebraic relations between the solutions of equation (2.6). On this ideal, we can define a action of an element $G \in G L_{n}(\mathbb{C})$ by the right multiplication on the matrix $x$ and $d \longrightarrow d \operatorname{det}(G)^{-1}$. The Galois group is now defined as

$$
G a l_{\mathbb{C}(t)}=\left\{G \in G L_{n}(\mathbb{C}): G \operatorname{Inv}=\operatorname{Inv}\right\} .
$$

Through this definition, we see immediately that $G$ is an algebraic Lie group, and, thus, (at least in small dimension) $G$ should belong to finitely many possible categories of groups. This is the base of the Kovacic algorithm to compute this group.

\section{A 2-dimensional example}

Let us consider the following example:

$$
\dot{X}=\left(\begin{array}{cc}
-\frac{t}{1-t^{2}} & \frac{1}{1-t^{2}} \\
1 & 0
\end{array}\right) X
$$

The fundamental matrix can here be computed explicitly and is given by

$$
\frac{1}{2}\left(\begin{array}{cc}
\left(1-t^{2}\right)^{-1 / 2}\left(e^{\arcsin (t)}+e^{-\arcsin (t)}\right) & \left(1-t^{2}\right)^{-1 / 2}\left(e^{\arcsin (t)}-e^{-\arcsin (t)}\right) \\
e^{\arcsin (t)}-e^{-\arcsin (t)} & e^{\arcsin (t)}+e^{-\arcsin (t)}
\end{array}\right) .
$$

From this, we can compute explicitly the invariant ideal. The determinant of the fundamental matrix is $2 / \sqrt{1-t^{2}}$. We, thus, obtain

$$
\operatorname{Inv}=\left\langle 4 d^{2}+t^{2}-1,2 x_{1,1} d-x_{2,2}, 2 d x_{1,2}-x_{2,1}, 2 d\left(x_{1,1}+x_{1,2}\right)\left(x_{2,1}-x_{2,2}\right)+1\right\rangle .
$$


This encodes a 1D variety over the base field $\mathbb{C}(t)$. Now, we can compute the Galois group and, thus, find

$$
G=\left\langle\left(\begin{array}{ll}
\frac{s}{2}+\frac{1}{2 s} & \frac{s}{2}-\frac{1}{2 s} \\
\frac{s}{2}-\frac{1}{2 s} & \frac{s}{2}+\frac{1}{2 s}
\end{array}\right)_{s \in \mathbb{C}^{*}},\left(\begin{array}{cc}
-1 & 0 \\
0 & -1
\end{array}\right)\right\rangle .
$$

This is a 1D group, and has two connected components. This is related to the fact that to express the solutions, we had to use one square root and one exponential-integral. This group is isomorphic to $\mathbb{Z} / 2 \mathbb{Z} \ltimes \mathbb{C}^{*}$ and, thus, solvable. The solvability of $G$ implies that the solutions can be represented explicitly.

Remark that we did not give at all a usable approach to compute the Galois group. Indeed, in practice, we want to compute the Galois group without having the fundamental matrix at first.

\subsection{Introduction to Morales-Ramis theorem}

\subsubsection{The Morales-Ramis theorem}

The main idea of the Morales-Ramis theorem is that if a Hamiltonian system is integrable, then the linearized system along a particular solution should also be "integrable." In this section, the Hamiltonian $H$ will only be assumed to be an $n$ degrees of freedom Hamiltonian over a general $2 n$-dimensional complex analytic manifold $\mathcal{M}$.

Let us consider a holomorphic function $f$ on $\mathcal{M}$, and a point $x \in \mathcal{M}$. The initial form of $f$ at $x$ is the lowest order nonzero term in the Taylor expansion of $f$ at $x$. It is, in particular, a homogeneous polynomial. If $f$ is a meromorphic function on $\mathcal{M}$, then its initial form is defined as the quotient of the initial form of its numerator and denominator.

This definition can then be generalized to curves. Given a complex analytic curve $\Gamma \subset \mathcal{M}$ parametrized by $t$, we consider for a holomorphic $f$ the Taylor expansion of $f$ at $x(t)$. The coefficients of this expansion are functions of $t$, and the initial form is the lowest order nonzero term (as a function of $t$ ) in this expansion. Remark that the valuation of $f$ at $x(t)$ can differ for some exceptional values of $t$ (this typically occurs at singular points of the variational equation). In the general meromorphic case, the initial form of $f$ on $\Gamma$ is then a homogeneous rational fraction with coefficients depending on $t$.

Lemma 3.1 (Ziglin [24]). Let $f_{1}, \ldots, f_{k}$ be germs of functionally independent meromorphic functions over a neighborhood of 0 in $\mathbb{C}^{n}$. Then, there exist polynomials $P_{1}, \ldots, P_{k} \in \mathbb{C}\left[z_{1}, \ldots, z_{n}\right]$ such that the initial forms at the origin of the functions $g_{i}=$ $P_{i}\left(f_{1}, \ldots, f_{k}\right)$ are meromorphic functions functionally independent in $\mathbb{C}\left(z_{1}, \ldots, z_{k}\right)$. 
Let us consider $\Gamma \subset \mathcal{M}$ a trajectory of the Hamiltonian field $X_{H}$. If this field has $n$ independent first integrals $f_{1}, \ldots, f_{n}$, then after possibly algebraic transformations, the initial forms of these first integrals can be assumed to be independent thanks to Ziglin lemma. Remark that if the Poisson bracket $\left\{f_{i}, f_{j}\right\}=0$, then the same is also true for their initial forms.

Theorem 3.2 (Morales-Ramis [14]). Let $H$ be a Hamiltonian over a complex analytic symplectic manifold $\mathcal{M}$ of dimension $2 n$. Assume that $H$ is integrable in the Liouville sense $\left(X_{H}\right.$ admits $n$ independent meromorphic first integrals, pairwise Poisson commuting). Let $\Gamma$ be a connected not reduced to a point particular solution of the Hamiltonian field $X_{H}$. Then, the identity component of the Galois group of the variational equation near $\Gamma$ is Abelian over the base field of rational functions on $\Gamma$.

\subsubsection{Homogeneous potentials}

In the case of homogeneous potentials, if we take for $\Gamma$ a curve coming from a Darboux point, the variational equation admits a particular form

$$
z(z-1) \ddot{X}+\left(\frac{3 k-2}{2 k} z-\frac{k-1}{k}\right) \dot{X}-\frac{\lambda}{2 k^{2}} X=0 .
$$

This equation is a hypergeometric equation and the Galois groups for all parameters values have been computed [10]. The result is that the integrability condition coming from the Morales-Ramis theorem becomes much more explicit

Theorem $3.3([5,15])$. Let $V$ be a homogeneous rational potential of homogeneity degree $k \in \mathbb{Z}^{*}$ and c a Darboux point with multiplier $k$. Assume that $\nabla^{2} V(c)$ (the Hessian matrix according to derivations in $q$ ) is diagonalizable. If $V$ has $n$ meromorphic first integrals which are in involution and functionally independent, then for any $\lambda \epsilon$ $\operatorname{Spect}\left(\nabla^{2} V(c)\right)$, the couple $(k, \lambda)$ belongs to the table

\begin{tabular}{rlrl}
\hline $\boldsymbol{k}$ & $\lambda$ & $\boldsymbol{k}$ & $\boldsymbol{\lambda}$ \\
\hline $\mathbb{Z}^{*}$ & $\frac{1}{2} i k(i k+k-2)$ & -3 & $-\frac{25}{8}+\frac{1}{8}\left(\frac{6}{5}+6 i\right)^{2}$ \\
$\mathbb{Z}^{*}$ & $\frac{1}{2}(i k+k-1)(i k+1)$ & -3 & $-\frac{25}{8}+\frac{1}{8}\left(\frac{12}{5}+6 i\right)^{2}$ \\
2 & $\mathbb{C}$ & 3 & $-\frac{1}{8}+\frac{1}{8}(2+6 i)^{2}$ \\
-2 & $\mathbb{C}$ & 3 & $-\frac{1}{8}+\frac{1}{8}\left(\frac{3}{2}+6 i\right)^{2}$ \\
-5 & $-\frac{49}{8}+\frac{1}{8}\left(\frac{10}{3}+10 i\right)^{2}$ & 3 & $-\frac{1}{8}+\frac{1}{8}\left(\frac{6}{5}+6 i\right)^{2}$ \\
-5 & $-\frac{49}{8}+\frac{1}{8}(4+10 i)^{2}$ & 3 & $-\frac{1}{8}+\frac{1}{8}\left(\frac{12}{5}+6 i\right)^{2}$ \\
-4 & $-\frac{9}{2}+\frac{1}{2}\left(\frac{4}{3}+4 i\right)^{2}$ & 4 & $-\frac{1}{2}+\frac{1}{2}\left(\frac{4}{3}+4 i\right)^{2}$ \\
-3 & $-\frac{25}{8}+\frac{1}{8}(2+6 i)^{2}$ & 5 & $-\frac{9}{8}+\frac{1}{8}\left(\frac{10}{3}+10 i\right)^{2}$ \\
-3 & $-\frac{25}{8}+\frac{1}{8}\left(\frac{3}{2}+6 i\right)^{2}$ & 5 & $-\frac{9}{8}+\frac{1}{8}(4+6 i)^{2}$ \\
\hline
\end{tabular}


Remark that the eigenvalues $k(k-1)$ always appear in the spectrum of $\left.\nabla^{2} V(c)\right)$ due to the fact that $c$ is always an eigenvector of $\nabla^{2} V(c)$ ), and through the Euler formula for homogeneous functions, we can prove that $\left.\nabla^{2} V(c)\right) \cdot c=k(k-1) c$. So this theorem gives generically $n-1$ integrability condition at each Darboux point.

With this, it becomes possible to make explicit the test for integrability, even for potentials with parameters (see $[12,13,21])$. However, two problems remain

- If the variational equation has a virtually Abelian Galois group, then the integrability status of the potential remains unknown.

- In the homogeneous potential case, even if several orbits can be found in theory, one needs to solve the Darboux point equation. This can be challenging even for a few number of variables: we know that there are probably a lot of solutions, so a lot of integrability conditions, so a low probability that the potential be integrable, but we are not able to compute these Darboux points! This is the typical problem in celestial mechanics $[17,22]$.

Thus, it is sometimes useful to have additional integrability conditions for a given orbit.

\subsubsection{Higher variational equations}

We now define higher variational equations, following Morales-Ramis-Simo [16, p. 860]. Let us denote the flow of the Hamiltonian field $X_{H}$ as $\varphi_{t}$. We denote

$$
\varphi_{t}(y)=\sum_{k} \varphi_{t}^{(k)}(x)(y-x)^{k}
$$

as the series expansion of $\varphi_{y}$ at $x$. We define accordingly

$$
X_{H}(y)=\sum_{k} X_{H}^{(k)}(x)(y-x)^{k} .
$$

The variational equations can now be written in a compact form

$$
\begin{gathered}
\dot{\varphi}_{t}^{(1)}=X_{H}^{(1)} \varphi_{t}^{(1)} \\
\dot{\varphi}_{t}^{(2)}=X_{H}^{(1)} \varphi_{t}^{(2)}+X_{H}^{(2)}\left(\varphi_{t}^{(1)}\right)^{2} \\
\dot{\varphi}_{t}^{(3)}=X_{H}^{(1)} \varphi_{t}^{(3)}+2 X_{H}^{(2)}\left(\varphi_{t}^{(1)}, \varphi_{t}^{(2)}\right)+X_{H}^{(3)}\left(\varphi_{t}^{(1)}\right)^{3},
\end{gathered}
$$

and the general formula is given by

$$
\dot{\varphi}_{t}^{(k)}=\sum_{j=1}^{k} \sum \frac{j !}{m_{1} ! \cdots m_{s} !} X_{H}^{(j)}\left(\left(\varphi_{t}^{\left(i_{1}\right)}\right)^{m_{1}}, \ldots,\left(\varphi_{t}^{\left(i_{s}\right)}\right)^{m_{s}}\right) .
$$

The point derivation corresponds to the derivation with respect to time along a particular solution $\Gamma$ of $X_{H}$. 
If the Hamiltonian system admits a first integral $f$, then the first-order variational equation admits a rational first integral, the initial form of $f$. The same holds for higher variational equations. Noting $f_{k}$ as the series expansion of $f$ consisting of the $k$ first terms of the Taylor series of $f$ beginning by the first nonzero term, we obtain for $f_{k}$ a polynomial (or rational fraction when $f$ is rational) which is a first integral of the $k$-th-order variational equation.

Remark that the first-order variational equation is a linear one, but higher order ones are not. These, however, can be "linearized" by the following process:

- The right term of each equation in $\varphi_{t}^{(k)}$ is a polynomial in $\varphi_{t}^{(i)}$ with $i<k$. So the right-hand side of the equation is a linear combination of monomial in $\varphi_{t}^{(i)}$ with $i<k$.

- A product of a solution of a linear differential equation is itself a solution of a linear differential equation (called symmetric power/product).

- We can, thus, replace each monomial of the right-hand side by a new unknown function, which will be a solution of a linear differential equation

The $k$-th-order variational equation (nonlinear version) can then be replaced by a linear differential system, whose solutions are the same as the linear version.

With the $k$-th variational equation, we can associate a Galois group. This Galois group preserves all rational invariants of the differential system and, in particular, the rational invariants coming from the first integrals of the Hamiltonian field. Through this process comes the following constraint on these Galois groups.

Theorem 3.4 (Morales-Ramis-Simo [16]). Let H be a Hamiltonian over a complex analytic symplectic manifold $M$ of dimension $2 n$. Assume that $H$ is integrable in the Liouville sense $\left(X_{H}\right.$ admits $n$ independent meromorphic first integrals, pairwise Poisson commuting). Let $\Gamma$ be a connected but not reduced to a point particular solution of $X_{H}$. Then, the identity component of the Galois group of the $k$-th-order variational equation near $\Gamma$ is Abelian over the base field of rational functions on $\Gamma$.

\section{Example}

This example of potential comes from [3]:

$$
V=\frac{1}{r}\left(1+3 \frac{q_{2}^{2}}{q_{1}^{2}}+\frac{101}{12} \frac{q_{2}^{4}}{q_{1}^{4}}\right),
$$

where $r^{2}=q_{1}^{2}+q_{2}^{2}$. The point $q_{1}=1, q_{2}=0, r=1$ is a Darboux point with the multiplier -1 . The eigenvalue computation gives the spectrum $\{2,5\}$ which is compatible with integrability as given by the Morales-Ramis theorem. Let us try to compute higher variational equations. We compute the series expansion of $V$ at order 2:

$$
V\left(1+q_{1}, q_{2}\right)=1-q_{1}+q_{1}^{2}+\frac{5}{2} q_{2}^{2}-q_{1}^{3}-\frac{15}{2} q_{1} q_{2}^{2}+O\left(\left\|q_{1}, q_{2}\right\|^{4}\right) .
$$


The second-order terms will lead to the first-order variational equation. The secondorder variational equation is built the following way:

$$
\ddot{X}_{1}=\phi^{-3} 2 X_{1}-\phi^{-4} 3 X_{1}^{2}, \quad \ddot{X}_{2}=\phi^{-3} 5 X_{2}-\phi^{-4} 15 X_{1} X_{2} \text {. }
$$

This equation is a nonlinear equation which is "linearized." New unknowns are introduced $y_{n_{1}, n_{2}, n_{3}, n_{4}}$ (here $1 \leq \sum n_{i} \leq 2$ ). In the following way, $y_{n_{1}, n_{2}, n_{3}, n_{4}}=$ $\dot{X}_{1}^{n_{1}} \dot{X}_{2}{ }^{n_{2}} X_{1}^{n_{3}} X_{2}^{n_{4}}$. We differentiate this expression and simplify it using the relation (3.1). This produces the following system:

$$
\dot{Y}=\left(\begin{array}{cccccccccc}
\ddot{X}_{1}=\phi^{-3} 2 X_{1}-\phi^{-4} 3 y_{2,0,0,0}, & \ddot{X}_{2}=\phi^{-3} 5 X_{2}-\phi^{-4} 15 y_{1,1,0,0} \\
0 & 2 & 0 & 0 & 0 & 0 & 0 & 0 & 0 & 0 \\
2 \phi^{-3} & 0 & 1 & 0 & 0 & 0 & 0 & 0 & 0 & 0 \\
0 & 4 \phi^{-3} & 0 & 0 & 0 & 0 & 0 & 0 & 0 & 0 \\
0 & 0 & 0 & 0 & 1 & 1 & 0 & 0 & 0 & 0 \\
0 & 0 & 0 & 5 \phi^{-3} & 0 & 1 & 0 & 0 & 0 & 0 \\
0 & 0 & 0 & 2 \phi^{-3} & 0 & 0 & 1 & 0 & 0 & 0 \\
0 & 0 & 0 & 0 & 2 \phi^{-3} & 5 \phi^{-3} & 0 & 0 & 0 & 0 \\
0 & 0 & 0 & 0 & 0 & 0 & 0 & 0 & 2 & 0 \\
0 & 0 & 0 & 0 & 0 & 0 & 0 & 5 \phi^{-3} & 0 & 1 \\
0 & 0 & 0 & 0 & 0 & 0 & 0 & 0 & 10 \phi^{-3} & 0
\end{array}\right) Y,
$$

$Y=\left(y_{2,0,0,0}, y_{1,0,1,0}, y_{0,0,2,0}, y_{1,1,0,0}, y_{1,0,0,1}, y_{0,1,1,0}, y_{0,0,1,1}, y_{0,2,0,0}, y_{0,1,0,1}, y_{0,0,0,2}\right)^{\top}$.

This $10 \times 10$ matrix is the matrix of the second symmetric power of the first variational equation. As shown in this example, the variational equations very fast become a very large size system of differential equations. They possess, however, a very important structure: they can be solved iteratively. Once the first-order variational equation is solved, the higher order ones can be solved through the variation of constant. In the case above, the first-order variational equation has a Galois group $\mathbb{C}^{+}$, and, in particular, a basis of solutions can be written as $P, P Q$, where $P \in \mathbb{C}(t)$ and $\dot{Q} \in \mathbb{C}(t)$. The function $Q$ is a transcendental function, an antiderivative, which corresponds to the fact that the Galois group is additive 1D. Now we can solve the second-order variational equation in the following way:

- The solutions of the $10 \times 10$ system are simply all products between the solutions of the first-order variational equation.

- These solutions are injected in the $X$ differential system. This produces a nonhomogeneous linear differential system with nonhomogeneous terms in $\mathbb{C}(t)[Q]$.

- If the solutions $X$ still belong to $\mathbb{C}(t)[Q]$, then the Galois group of the second-order variational equation will still be $1 \mathrm{D}$, so equal to $\mathbb{C}^{+}$. This can be tested automatically while solving in rational functions a linear differential system.

- If the search of such a solution fails, then a constraint on the monodromy of the solutions is searched.

After reparametrization, the function $Q$ here can be chosen as $\operatorname{arctanh}\left(t^{-1}\right)$. We just apply the following lemma: 
Lemma 3.5 ([4]). Let $F \in \mathbb{C}\left(z_{1}\right)\left[z_{2}\right]$ and

$$
f(t)=F\left(t, \operatorname{arctanh}\left(\frac{1}{t}\right)\right) \in \mathbb{C}(t)\left[\operatorname{arctanh}\left(\frac{1}{t}\right)\right] .
$$

We consider the following differential field extension and its differential Galois group:

$$
K=\mathbb{C}\left(t, \operatorname{arctanh}\left(\frac{1}{t}\right), \int f d t\right), \quad G=\operatorname{Gal}_{\text {diff }}(K / \mathbb{C}(t)) .
$$

If $G$ is Abelian, then

$$
\frac{\partial}{\partial \alpha} \operatorname{Res} F\left(t, \operatorname{arctanh}\left(\frac{1}{t}\right)+\alpha\right)=0 \quad \forall \alpha \in \mathbb{C},
$$

where Res corresponds to the residue.

Applying this procedure allows us to prove that the fifth variational equation has not a virtually Abelian Galois group.

\subsection{Application to parametrized potentials}

\subsubsection{Space of germs of integrable potentials}

Let us consider a homogeneous potential of degree $k$ with the series expansion

$$
V\left(1+q_{1}, q_{2}, \ldots, q_{n}\right)=k q_{1}+\sum_{i=1}^{n} \frac{\lambda_{i}}{2} q_{i}^{2}+O\left(\|q\|^{3}\right) .
$$

Assume that the integrability condition on the $\lambda_{i}$ is satisfied. Remark that any potential satisfying the Morales Ramis integrability condition can be reduced under this form. What are the integrability conditions on higher order terms?

Let us denote $E_{m, \lambda_{1}, \ldots, \lambda_{n}}$ the affine space of series expansions of homogeneous potentials of the form 4.1 of order $m$. We denote

$$
V\left(1+q_{1}, q_{2}, \ldots, q_{n}\right)=q_{1}^{k}\left(1+\sum_{i=1}^{n} \frac{\lambda_{i}}{2}\left(\frac{q_{i}}{q_{1}}\right)^{2}+\sum u_{i_{1}, \ldots, i_{n}} \prod\left(\frac{q_{j}}{q_{1}}\right)^{i_{j}}\right)
$$

as a parametrization of the space $E_{m, \lambda_{1}, \ldots, \lambda_{n}}$. Remark that we have fixed the eigenvalues $\lambda_{1}, \ldots, \lambda_{n}$. Using only the series expansion up to order $m$, we can compute the variational equation of order $m-1$. The solutions of this $(m-1)$ th-order variational equation will depend on the coefficients $u$. But in a specific way:

Proposition 4.1. Let us consider the homogeneous potential $V$ given by (4.1) with fixed eigenvalues $\lambda_{1}, \ldots, \lambda_{n}$. Then, the fundamental matrix of the $(m-1)$ th-order variational equation is an affine function of the highest order derivatives $u_{i_{1}, \ldots, i_{n}}, i_{1}+\cdots+i_{n}=$ $m$. More generally, it is a polynomial in $u$ of weighted degree $\leq m-2$ with weights $\operatorname{deg}\left(u_{i_{1}, \ldots, i_{n}}\right)=i_{1}+\cdots+i_{n}-2$. 
Remark that a weighted degree is completely defined by its value on $u_{i_{1}, \ldots, i_{n}}$ : we can deduce the degree of monomials (just by addition) and then polynomials (taking the maximum of the degrees of the monomials).

Proof. Let us first remark that the first result is included in the second. The degree of $u_{i_{1}, \ldots, i_{n}}, i_{1}+\cdots+i_{n}=m$ is by definition $m-2$. So any monomial containing such highest order derivative cannot contain anything else (as the total degree is $\leq m-2$ ). So this means that the fundamental matrix is an affine function of the highest order derivatives.

The $(m-1)$ th-order variational equation matrix can be written as follows:

$$
\left(\begin{array}{ccccc}
A & B_{1,2} & B_{1,3} & \ldots & B_{1, m-1} \\
0 & \operatorname{Sym}^{2}(A) & B_{2,3} & \ldots & B_{2, m-1} \\
0 & 0 & \ldots & \ldots & \ldots \\
0 & \ldots & 0 & \operatorname{Sym}^{m-2}(A) & \ldots \\
0 & \ldots & & 0 & \operatorname{Sym}^{m-1}(A)
\end{array}\right),
$$

where $A$ is the matrix of the first-order variational equation. This matrix is upper block triangular, and the matrices $\operatorname{Sym}^{i}(A)$ (symmetric powers of $A$ ) do not depend on any parameters (as the eigenvalues $\lambda_{i}$ are fixed). So the only parameters of this system are the $u_{i_{1}, \ldots, i_{n}}$, which only appear in the matrices $B_{j, l}$. Following the procedure for building higher variational equations, we have that $B_{j, l}$ is linear in the derivatives $u_{i_{1}, \ldots, i_{n}}$, with $i_{1}+\cdots+i_{n}=l-j+2$.

This system can be solved through the variation of constants and the parameters $u_{i_{1}, \ldots, i_{n}}$ only appear in the nonhomogeneous part linearly. So the solutions of the variational equations depend polynomially on the $u$. Let us now look at the weighted degree of these polynomials. Using this block structure of the variational equation, we cut the components of a solution in $m-1$ parts, the last being associated with the matrix block $\operatorname{Sym}^{m-1}(A)$.

Lemma 4.2. The weighted degree of the l-th part of the components of a solution of (4.2) is $\leq m-1-l$.

Proof. This is done by recurrence. For the solution components of the last block (with $\operatorname{Sym}^{m-1}(A)$ ), they are the solutions of a homogeneous systems with no parameters. So that they are of weighted degree 0 . This proves the lemma for $l=m-1$.

Assume that the lemma is true for all $s \geq l+1$ for some fixed $l$. Let us prove it for $s=l$. The part $l$ components $X_{l}$ are the solutions of a system of the form

$$
\dot{X}_{l}-\operatorname{Sym}^{l}(A) X_{l}=B_{l, l+1} X_{l+1}+\cdots+B_{l, m-1} X_{m-1} .
$$

The weighted degree of $B_{l, s}$ is $s-l$ and, by recurrence assumption, the weighted degree of $X_{s}$ is $s \geq l+1$ is $\leq m-1-s$. So the nonhomogeneous part has at most a weighted 
degree of

$$
\max _{s=l+1, \ldots, m-1} s-l+m-1-s=m-1-l .
$$

The lemma applied to a basis of solutions gives that the maximal weighted degree is $m-2$.

This suggests to compute these higher variational equation constraints iteratively. Moreover, the integrability constraints on the Galois group of these variational equations will then be polynomial in the higher order derivatives $u_{i_{1}, \ldots, i_{n}}$. When searching (possibly) integrable potentials having a series expansion in $E$, we obtain at each step (among other lower derivatives constraints) linear constraints of the highest order derivatives. If these constraints are strong enough at all orders, this approach can lead to uniqueness theorems.

Lemma 4.3 (Combot [3]). Let $V_{1}$ and $V_{2}$ be two rational integrable homogeneous potentials on $\mathbb{C}^{2}$ of degree -1 with a Darboux point of the form $c=(1,0)$ with multiplier -1 . Assume that there exists $k_{0} \geq 2$ such that the integrability constraint of $\left(V E_{k}\right)$ is nondegenerate $\forall k \geq k_{0}$ (see [3] for definition). If

$$
\frac{\partial^{i+j}}{\partial q_{1}^{i} \partial q_{2}^{j}} V_{1}(c)=\frac{\partial^{i+j}}{\partial q_{1}^{i} \partial q_{2}^{j}} V_{2}(c) \quad \forall(i, j) \text { such that } i+j \leq k_{0},
$$

then $V_{1}=V_{2}$.

Another consequence is the possible computation of the space of integrable series expansion of order $m$ in $E$. The following result was obtained by Combot [6] for $k=$ $-1, \lambda_{1}=2, \lambda_{2}=5, \lambda_{3}=44, m \leq 4$ :

$$
\begin{array}{ll}
m=2 & q_{1}^{-1}\left(1+\frac{5}{2} \frac{q_{2}^{2}}{q_{1}^{2}}+\frac{44}{2} \frac{q_{3}^{2}}{q_{1}^{2}}+O\left(\frac{\left(q_{2}, q_{3}\right)^{3}}{q_{1}^{3}}\right)\right) \\
m=3 & q_{1}^{-1}\left(1+\frac{5}{2} \frac{q_{2}^{2}}{q_{1}^{2}}+\frac{44}{2} \frac{q_{3}^{2}}{q_{1}^{2}}+u_{3,1} \frac{q_{2}^{2} q_{3}}{q_{1}^{3}}+O\left(\frac{\left(q_{2}, q_{3}\right)^{4}}{q_{1}^{4}}\right)\right) \\
m=4 & q_{1}^{-1}\left(1+\frac{5}{2} \frac{q_{2}^{2}}{q_{1}^{2}}+\frac{44}{2} \frac{q_{3}^{2}}{q_{1}^{2}}+u_{3,1} \frac{q_{2}^{2} q_{3}}{q_{1}^{3}}+\left(\frac{7}{459} u_{3,1}^{2}+\frac{175}{24}\right) \frac{q_{2}^{4}}{q_{1}^{4}}+\right. \\
& \left.\left(-\frac{734}{7775} u_{3,1}^{2}+\frac{130,779}{622}\right) \frac{q_{2}^{2} q_{3}^{2}}{q_{1}^{4}}+\frac{682,538,736}{1,082,611} \frac{q_{3}^{4}}{q_{1}^{4}}+O\left(\frac{\left(q_{1}, q_{3}\right)^{5}}{q_{1}^{5}}\right)\right) .
\end{array}
$$

For $m \geq 5$, no integrable potential of this form remains. In general, the variety of the integrable series expansion of order $m-1$ in $E$ is an algebraic variety. If some kind of nondegeneracy condition on higher variational equation holds, the Hilbert dimension of this variety decreases with $m$. This process always seems to lead to all integrable potentials for some particular set of eigenvalues. For the set $k=-1,\left\{\lambda_{1}, \lambda_{2}, \lambda_{3}\right\}=$ $\{2,5,44\}$, this allows us to conclude: 
Corollary 4.4 (Combot [6]). A rational homogeneous potential of degree -1 of dimension 3 with a Darboux point with the multiplier -1 and the eigenvalue set $\{2,5,44\}$ is not integrable.

This combination of eigenvalues was possible in the Morales-Ramis theorem, but the use of higher variational equations and the Morales-Ramis-Simo theorem allowed us to remove this combination from the possible integrable potentials. This approach can now be effective even in cases where only bounding of the trace of the Hessian matrix at Darboux points can be obtained.

Let us consider a family of homogeneous potentials $W$ of degree $k$. Assume that we know a bound $B \in \mathbb{N}$ such that

$$
\forall V \in W, \exists c \text { Darboux point with multiplier } k, \operatorname{tr}\left(\nabla^{2} V(c)\right) \leq B \text {. }
$$

This property was called "bounded eigenvalue property" in [7]. Then, integrable potentials in $W$ are included in a finite union of affine spaces of type $E$. The study of higher variational equations integrability conditions for each affine space $E$ allows us to find strong integrability conditions for potentials in $W$, and hopefully to prove that no unknown integrable potentials rely on these spaces $E$.

\subsubsection{Eigenvalue bounding of some $n$-body problems}

The $n$-body problem is famous for having a difficulty to solve Darboux point equations (which are called in this specific topic central configuration). Indeed, even finiteness is not clear $[1,11,20]$. Here, the need of information of the spectrum of the Hessian of $V$ at these points is even more.

Theorem 4.5 (Combot [6]). We consider the collinear four-body problem with positive masses. The potential after reduction is a 3D potential homogeneous of degree -1. Let $c$ be the real Darboux point with multiplier -1 (existence and uniqueness up to translation due to [18]). Then, $\operatorname{tr}\left(\nabla^{2} V(c)\right)<70$.

This theorem comes down to a real algebraic problem, and such a bound can be automatically proved by real algebra software such as RAGLib [9]. Looking at the MoralesRamis table, we see that for $k=-1$, the minimal possible eigenvalue is -1 and the set is discrete. Thus, only finitely many eigenvalue sets are possible. In this $n$-body problem, we have, moreover, a better lower bound.

Theorem 4.6 (Pacella [19]). We consider the collinear four-body problem potential with positive masses after reduction and the Darboux point $c$ with multiplier -1 . Then, $\left.\operatorname{Spect}\left(\nabla^{2} V(c)\right)\right)=\left\{2, \lambda_{1}, \lambda_{2}\right\}$ with $\lambda_{1}, \lambda_{2}>2$. 
Combining this with the upper bound of the trace, we obtain finitely many possible sets:

$$
\begin{array}{r}
\{5,5\},\{5,9\},\{5,14\},\{5,20\},\{5,27\},\{5,35\},\{5,44\},\{5,54\},\{9,9\}, \\
\{9,14\},\{9,20\},\{9,27\},\{9,35\},\{9,44\},\{9,54\},\{14,14\},\{14,20\},\{14,27\}, \\
\{14,35\},\{14,44\},\{20,20\},\{20,27\},\{20,35\},\{20,44\},\{27,27\},\{27,35\} .
\end{array}
$$

For each eigenvalue set, a computation of the higher variational equations up to order 5 allows us to prove that these eigenvalue sets are not possible for integrability.

Corollary 4.7 (Combot [6]). The collinear four-body problem with positive masses is not integrable.

Acknowledgement I wish to thank the numerous valuable comments and suggestions of the anonymous referee.

\section{Bibliography}

[1] A. Albouy and V. Kaloshin. Finiteness of central configurations of five bodies in the plane. Annals of mathematics, 176:535-588, 2012.

[2] V. I. Arnol'd. Mathematical methods of classical mechanics, volume 60. Springer, 1989.

[3] T. Combot. Integrable homogeneous potentials of degree -1 in the plane with small eigenvalues. arXiv:1110.6130, 2011.

[4] T. Combot. Integrability conditions at order 2 for homogeneous potentials of degree -1 . Nonlinearity, 26, 2013.

[5] T. Combot. A note on algebraic potentials and Morales-Ramis theory. Celestial Mechanics and Dynamical Astronomy, pages 1-22, 2013.10.1007/s10569-013-9470-2.

[6] T. Combot. Integrability and non integrability of some $n$ body problems. Recent Advances in Celestial and Space Mechanics, 23:1-30, 2015.

[7] T. Combot and C. Koutschan. Third order integrability conditions for homogeneous potentials of degree -1. Journal of Mathematical Physics, 53(8):082704, 2012.

[8] T. Combot. Non-integrability of a self-gravitating riemann liquid ellipsoid. Regular and Chaotic Dynamics, 18(5):497-507, 2013.

[9] M. Safey El Din. Raglib: A library for real algebraic geometry. http://www-calfor.lip6.fr/ safey/ RAGLib/, 2003.

[10] T. Kimura. On Riemann's equations which are solvable by quadratures. Funkcialaj Ekvacioj, 12:269-281, 1970.

[11] T. L. Lee and M. Santoprete. Central configurations of the five-body problem with equal masses. Celestial Mechanics and Dynamical Astronomy, 104(4):369-381, 2009.

[12] A. J. Maciejewski and M. Przybylska. All meromorphically integrable 2D Hamiltonian systems with homogeneous potential of degree 3. Physics Letters A, 327(5-6):461-473, 2004.

[13] A. J. Maciejewski and M. Przybylska. Darboux points and integrability of Hamiltonian systems with homogeneous polynomial potential. Journal of Mathematical Physics, 46:062901, 2005.

[14] J. J. Morales-Ruiz and J. P. Ramis. Galoisian obstructions to integrability of hamiltonian systems. Methods and Applications of Analysis, 8(1):33-96, 2001.

[15] J. J. Morales-Ruiz and J.P. Ramis. A note on the non-integrability of some hamiltonian systems with a homogeneous potential. Methods and applications of analysis, 8(1):113-120, 2001. 
[16] J.J. Morales-Ruiz, J. P. Ramis, and C. Simó. Integrability of Hamiltonian systems and differential Galois groups of higher variational equations. Annales scientifiques de l'Ecole normale supérieure, 40(6):845-884, 2007.

[17] J. J. Morales-Ruiz and S. Simon. On the meromorphic non-integrability of some $\mathrm{N}$-body problems. Discrete and Continuous Dynamical Systems (DCDS-A), 24(4):1225-1273, 2009.

[18] F. R. Moulton. The straight line solutions of the problem of $n$ bodies. The Annals of Mathematics, 12(1):1-17, 1910.

[19] F. Pacella. Central configurations of the $\mathrm{n}$-body problem via equivariant morse theory. Archive for Rational Mechanics and Analysis, 97(1):59-74, 1987.

[20] E. Pina and P. Lonngi. Central configurations for the planar newtonian four-body problem. Celestial Mechanics and Dynamical Astronomy, 108(1):73-93, 2010.

[21] M. Przybylska. Finiteness of integrable n-dimensional homogeneous polynomial potentials. Physics Letters A, 369(3):180-187, 2007.

[22] A. Tsygvintsev. The meromorphic non-integrability of the three-body problem. Journal fuer die reine und angewandte Mathematik (Crelles Journal), 2001(537):127-149, 2001.

[23] H. Yoshida. A criterion for the non-existence of an additional integral in Hamiltonian systems with a homogeneous potential. Physica D: Nonlinear Phenomena, 29(1-2):128-142, 1987.

[24] S. L. Ziglin. Branching of solutions and nonexistence of first integrals in hamiltonian mechanics. I. Functional Analysis and Its Applications, 16(3):181-189, 1982. 